\title{
ARTICLE
}

\section{Determination of the primary X-ray spectrum using a PMMA wedge, a flat panel and the Monte Carlo method}

\author{
Sergio Gallardo ${ }^{\mathrm{a}^{*}}$, Belén Juste ${ }^{\mathrm{a}}$, Andrea Querol ${ }^{\mathrm{a}}$, Fausto Pozuelo ${ }^{\mathrm{a}}$, Gumersindo Verdúa José Ródenas $^{\mathrm{a}}$, Juan M. \\ Campayo $^{\mathrm{b}}$ and Sergio Díez \\ ${ }^{a}$ Universitat Politècnica de València, Camí de Vera s/n, 46022, València, Spain; ${ }^{b}$ Hospital Clínic Universitari de València, Avda. \\ Blasco Ibáñez, 17. 46010, València, Spain
}

\begin{abstract}
An accurate knowledge of the photon spectra emitted by X-ray tubes in radiodiagnostic is essential to better estimate the imparted dose to patients and to improve the quality image obtained with these devices. However, it is difficult to measure the energy spectrum of these tubes due to the pile up effect produced by the high fluence of X-ray beams. In this work, it is proposed the use of a flat panel detector together with a PMMA wedge to obtain a dose distribution for different high voltages. The relation between the dose curve recorded by the flat panel and the primary beam X-ray spectrum is defined by a Response matrix. The MCNP5 code based on the Monte Carlo method has been used to simulate the actual experimental acquisition including the X-ray tube, the PMMA wedge and a flat panel. A Response matrix has been obtained by means of different monochromatic beams. Knowing the dose distribution for a given conditions and the Response matrix, the primary spectrum can be obtained. The Modified Truncated Singular Value Decomposition (MTSVD) method has been applied to unfold primary spectra using Matlab (C) algorithms. Unfolded spectra are compared with theoretical X-ray spectra obtained from IPEM 78 catalogue report. Results show that the hybrid technique -experimental-Monte Carlo method- could represent a valid tool in order to complement the Quality Control of X-ray tubes in the radiodiagnostic energy range.
\end{abstract}

Keywords: Monte Carlo; flat panel; PMMA wedge; dose distribution; unfolding

\section{Introduction}

Obtaining X-ray spectra from radiodiagnostic X-ray units is a complex task mainly due to the high photon flux and the low energy of emitted particles by the tube. In X-ray spectra measurements, photon fluence near the detector needs to be limited, as detectors cease to work properly at high count rates. At a high fluence rate, a 'pile-up' effect can be observed, which distorts the Pulse Height Distribution (PHD). To obtain appropriate count rates, a small diameter collimator and a long tube-detector distance should be used. However, in general it is not possible due to the small space available in X-ray rooms. To reduce the high fluence reaching the detector, a dispersive or attenuating material is needed. In this paper, it is studied the potentiality of using a flat panel as detector and a PMMA wedge as attenuating material to estimate primary X-ray spectra. The basis of flat panels consists in transforming the absorbed X-rays into charge carriers. Depending on the physics applied to obtain the current, it can be found direct and indirect measurement devices. In direct devices, amorphous Selenium (a:Se) is normally used to directly transform

*Corresponding author. Email: sergalbe@upv.es
X-rays into current. In indirect devices, a scintillator material absorbs the X-rays and converts them into visible light photons that pass onto a photodiode array. A semiconductor foil is used to convert visible light photons to charge carriers. Normally amorphous Silicon is preferred due to its high rate for Detective Quantum Efficiency (DQE). The most common scintillators used in flat panel imaging in indirect measurements are Gadolinium Oxysulfide (GOS) and Thallium doped Cesium Iodide CsI(Tl).

In this work, it is proposed a hybrid technique -experimental-Monte Carlo method- to estimate primary X-ray spectra using a flat panel (GOS scintillator) and a PMMA wedge. The PMMA wedge is superposed between the X-ray focus and the flat panel. When the flat panel is irradiated, it registers an absorbed dose gradient due to the attenuation of X-rays produced in the wedge. For certain working conditions (high voltage, filter thickness and current) of X-ray tube, an absorbed dose curve is obtained. This curve is directly related to the primary spectrum by means of a Response function. In most applications, the Response function can be approximated by a matrix, which can be obtained using the Monte Carlo method. The MCNP5 code [1] has been used to calculate the Response matrix of the system by 
simulating several monoenergetic X-ray beams and obtaining the absorbed-dose curve for each one. When the dose curve and the Response matrix are known, to estimate the primary spectrum it is necessary to inverse the matrix. However, this process is extremely inefficient because it is an ill-posed problem and consequently the Response matrix is ill conditioned. It can be found in the literature different methods to unfold the primary spectrum, most of them based on the Singular Value Decomposition (SVD) techniques [2]. In this paper, it has been used the Modified Truncated Singular Value Decomposition (MTSVD) method [3] to unfold the primary X-ray spectrum knowing the Response matrix and the absorbed dose curve for some given conditions. The method has been tested simulating the dose curve for different X-ray spectra extracted from the IPEM 78 Report [4]. Once dose curves are obtained, the unfolding method is applied and a primary spectrum is calculated and compared with the theoretical (IPEM 78) one. The method has been validated using dose curves experimentally measured.

\section{Experimental equipment and measurements}

The experimental equipment is composed by a commercial X-ray tube (Toshiba mod E7299X), a flat panel (Hamamatsu C9312SK), a PMMA wedge $(12.5 \times 12.5 \times 10 \mathrm{~cm})$ and a laptop with the appropriate acquisition software. The C9312SK flat panel uses an indirect converting scintillator made of Gadolinium Oxysulfid, which is connected to the image sensor through a fiber optic plate. The pixel size is $50 \times 50 \mu \mathrm{m}^{2}$. $\mathrm{X}$-rays hit the $140 \mu \mathrm{m}$ thick scintillator through the entrance window of $1 \mathrm{~mm}$ thick carbon. The $3 \mathrm{~mm}$ thick fiber optic is located behind the scintillator. A shielding is provided by a thin copper foil. The distance between the flat panel surface and the X-ray focus was fixed at $60 \mathrm{~cm}$. A radiation field of $15 \times 15 \mathrm{~cm}^{2}$ was used. The PMMA wedge and the flat panel were irradiated at different working conditions varying high voltage and current of the X-ray tube. Measurements were made for the following voltages: $60,70,80,90$ and $100 \mathrm{kVp}$. A gray-scaled matrix $(2490 \times 2490)$ is obtained for each case. Selecting and collapsing a central band of the matrix a dose curve can be obtained. A central band has been chosen in order to minimize penumbra effects.

\section{The Monte Carlo model}

The MCNP5 model includes the X-ray focus, a PMMA wedge and the flat panel. The geometry of the MCNP5 model can be seen in Figure 1. The photon fluence has been measured in the GOS layer at 84 different positions along the $\mathrm{X}$ axis using a F4MESH tally [1]. Photon fluence has been converted into dose using the GOS energy-mass absorption coefficient $\left(\frac{\mu_{e n}}{\rho}\right)$ provided by the National Institute of Standards and Technology (NIST) [5]. As a result it is obtained an absorbed dose curve in $\mu$ Gy per emitted photon.

It has been simulated 500 million particles to obtain relative errors lower than $1.0 \%$. MODE P, E has been activated to follow tracks of photons and electrons. A cutoff of $5 \mathrm{keV}$ for electrons has been used in order to reduce the computation time. Theoretical X-ray spectra have been extracted from the IPEM 78 Report data base [4].

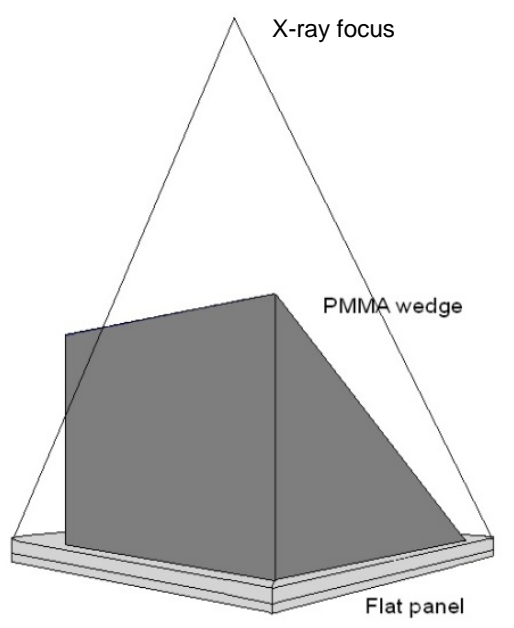

Figure 1. Geometry layout of the MCNP5 model.

To compare gradients of experimental and simulated doses, both curves have been normalized obtaining the same area under both curves. Results corresponding to $70 \mathrm{kVp}$ spectra are shown in Figure 2. The MCNP5 curve includes the $3 \sigma$ error bars. In general a good agreement between both gradients is achieved.

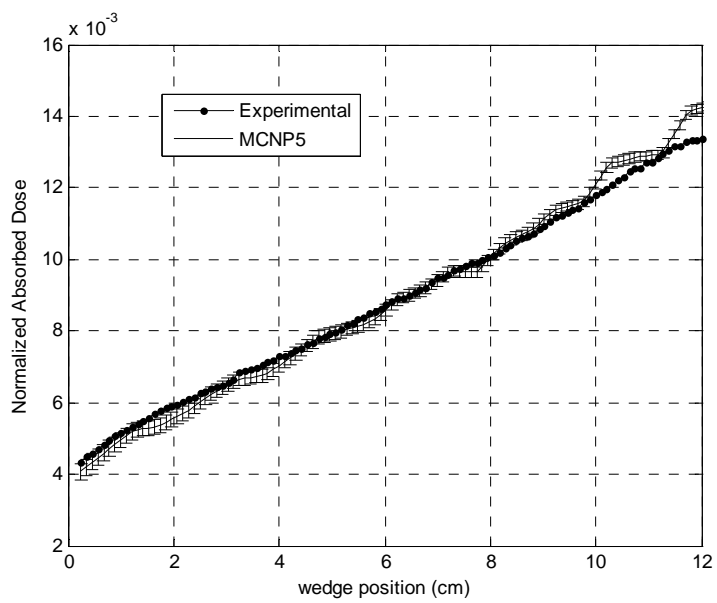

Figure 2. Normalized absorbed dose curves corresponding to MCNP5 and experimental measurement $70 \mathrm{kVp}$ spectrum).

\section{Unfolding methods}

The relation between the dose curve and the primary spectrum is defined by the Response matrix $(R)$, which can be obtained simulating several monochromatic 
photon beams and obtaining the corresponding dose curves:

$$
R \vec{s}=\vec{m}
$$

where

$\vec{s}$ is the unknown primary spectrum, and $\vec{m}$ is the dose curve registered.

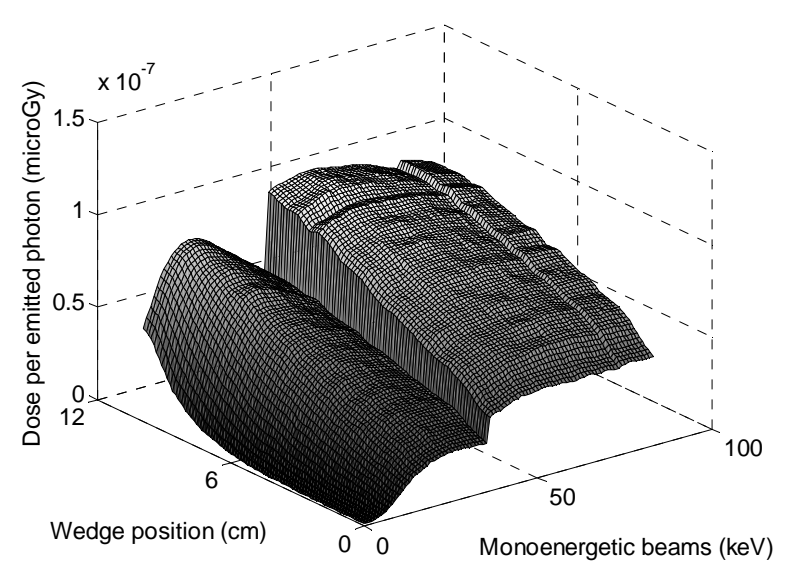

Figure 3. Response matrix.

In Figure 3 it is shown the Response matrix of the system. As it can be seen there is an abrupt change at 50 $\mathrm{keV}$ corresponding to the K-edge of GOS.

Once the Response matrix is known, the equation (1) theoretically permits to obtain the primary spectrum $\vec{S}$. But as the determination of this matrix is affected by some errors an approximation $\overrightarrow{\tilde{S}}$ to $\vec{S}$ is chosen in such a way that minimizes the 2-norm of the residual vector $\|R \overrightarrow{\tilde{s}}-\vec{m}\|_{2} \cdot R$ has a sparse structure with most of non-zero elements lying on the main diagonal of the matrix [3]. Furthermore, this matrix is ill conditioned (condition number of $R \quad 6.2 \cdot 10^{19}$ ). The singular values of the Response matrix $R$, are shown in Figure 4.

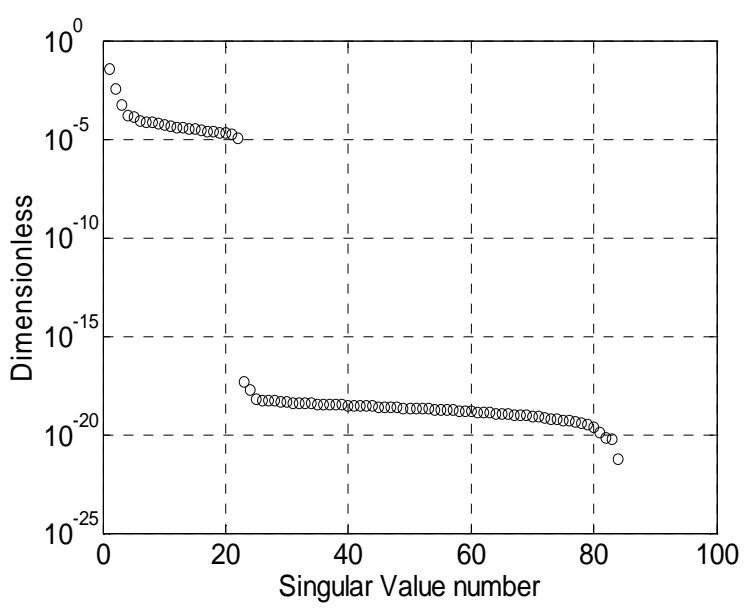

Figure 4. Singular Values of the Response matrix.

As it can be seen, singular values decay rapidly to 0 , when the last singular values are very small. These small singular values make matrix $R$ to be ill conditioned. Due to the fact that $R$ can be considered as rank deficient, there are a large number of solutions for the Least Squares problem $\|R \overrightarrow{\tilde{s}}-\vec{m}\|_{2}$. An optimum solution can be obtained, generating a new Response matrix, $R_{k}$, removing the parts of the solution corresponding to the smallest singular values $[3,6]$. In this way, the obtained vector is the solution of the minimization problem:

$$
\min \left\{\|\vec{s}\|_{2}\right\} \text { subject to } \min \left\{\left\|R_{k} \vec{s}-\vec{m}\right\|_{2}\right\}
$$

In the Modified Truncated Singular Value Decomposition (MTSVD), the 2-norm, $\|\vec{s}\|_{2}$, in the problem represented by eq. (2) is replaced by the seminorm $\left\|L_{P} \vec{S}\right\|_{2}$, where $L_{P}$ is a discrete approximation to the $\mathrm{p}$-th derivative operator [3]. In the Figure 5 it is shown the representation of the solution norm and the residual norm for different singular values (L-curve).

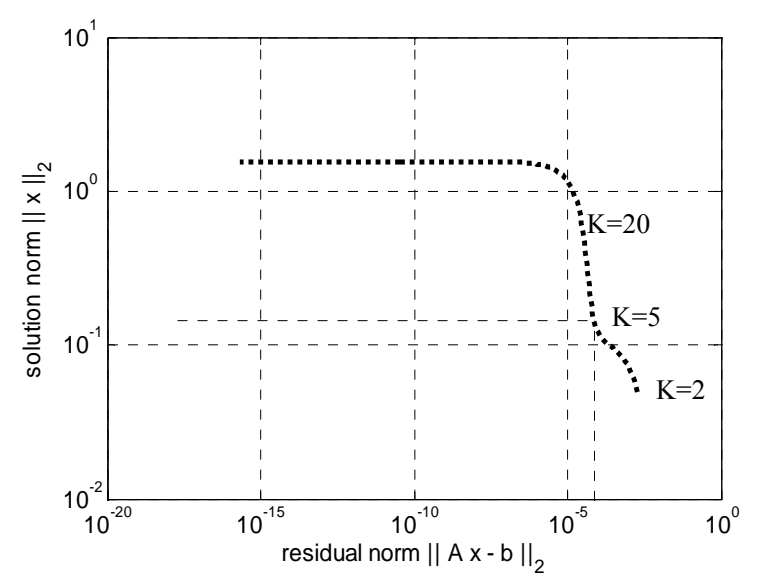

Figure 5. L-curve (corner marks the optimal $k$ value).

\section{Results and discussion}

The election of the truncation parameter, $k$ (number of singular values considered) is essential for an appropriate unfolding of the spectrum. In Figure 6 it is shown the $50 \mathrm{kVp}$ unfolded spectra when $k$ is varied.

When the number of singular values is too low, the unfolded spectrum has a poor resolution. However, for higher $k$ values, strong fluctuations and noise start to appear in the spectrum. The optimal $k$ corresponds with the corner of the L-curve. This value minimizes both the solution and the residual norms.

For different voltages, the dose curve has been obtained experimentally and by simulation. Using these curves and the Response matrix, unfolded spectra can be calculated corresponding to both experimental and simulated curves. Unfolded spectra have been compared with the theoretical spectrum, extracted from the IPEM 78 Report data base. $1 \mathrm{keV}$ resolution energy bin has been considered to obtain the Response matrix.

In Figure 7 it is shown a comparison between theoretical, experimental and simulated spectra 
corresponding to $60 \mathrm{kVp}$.

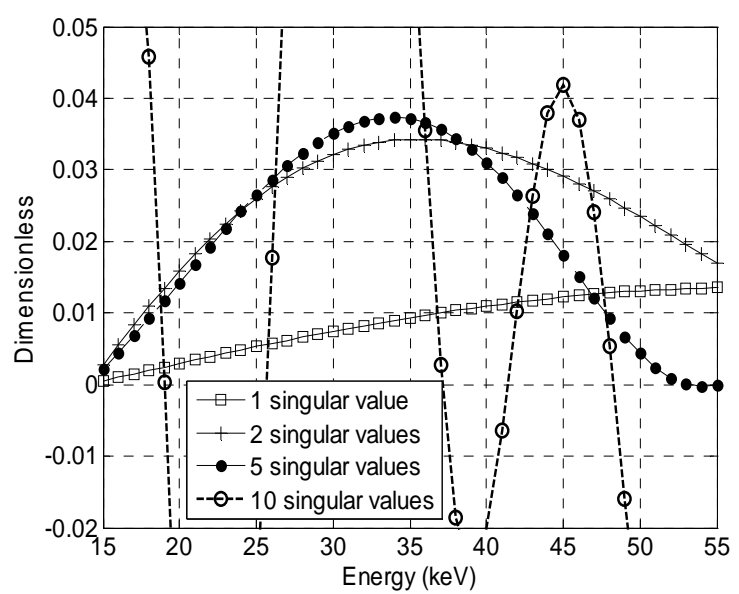

Figure 6. MTSVD Unfolded spectra $(50 \mathrm{kVp})$ for different number of singular values.

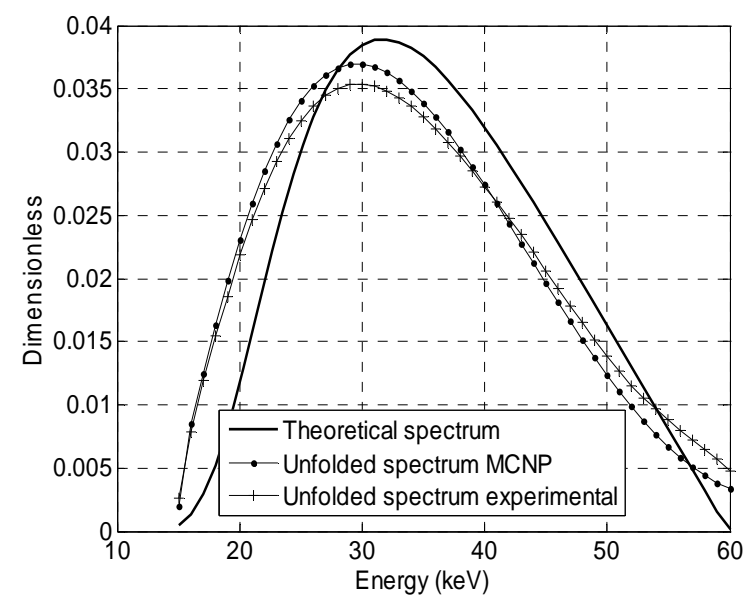

Figure 7. MTSVD Unfolded spectra $60 \mathrm{kVp}(k=5)$.

As it can be seen, in both cases the Bremsstrahlung continuous is slightly shifted respect to the theoretical distribution. The X-ray spectrum presents characteristic lines of Tungsten $\left(\mathrm{K}_{\alpha}, \mathrm{K}_{\beta}\right)$ when the voltage is higher than $70 \mathrm{kVp}$, as it can be seen in Figure 8. The method is not able to unfold the intensity of the characteristic lines, being them confused with the Bremsstrahlung continuous. Again, a shifted is observed between the reconstructed and theoretical spectra. Results show that the K-edge of GOS does not affect to the spectra reconstruction when MTSVD is used. The $\mathrm{L}_{\mathrm{p}}$ derivative operator is able to manage the K-edge abrupt change, smoothing the unfolded spectrum. The root mean square (RMS) has been calculated for each unfolded spectra respect to the IPEM 78 theoretical spectrum. In any case, the RMS is lower than $15 \%$.

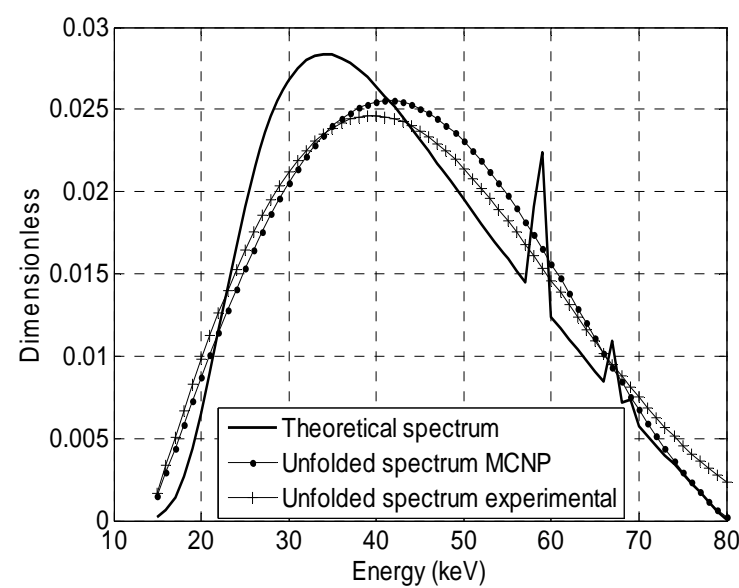

Figure 8. MTSVD Unfolded spectra $80 \mathrm{kVp}(k=5)$.

\section{Conclusion}

A hybrid technique -experimental-Monte Carlo method- using a flat panel and a PMMA wedge has been used to estimate primary X-ray spectra in the diagnostic energy range. With this goal, a Response matrix of the system is needed and it can be obtained using a MCNP5 model. This matrix is ill conditioned, and consequently, it is necessary to use unfolding methods to obtain a pseudoinverse matrix. It has been proved that the MTSVD method can be used with this aim. The optimal truncation parameter can be calculated using the L-curve criterion. The method permits to unfold the Bremsstrahlung continuous with acceptable results (RMS lower than $15 \%$ ), but is not able to unfold the characteristic lines of Tungsten with a proper resolution.

\section{References}

[1] X-5 MONTE CARLO TEAM, MCNP - A General Monte Carlo Nparticle Transport Code, Version 5 LA-UR-03-1987, Los Alamos National Laboratory, April, (2003).

[2] G. H. Golub and C. F. Van Loan, Matrix Computations, The Johns Hopkins University Press, Third edition, (1996).

[3] P. C. Hansen, T. Sekii and H. Shibabhashi, The modified truncated SVD method for regularization in general form, SIAM J. Sci. Comput. 13 (1992), pp. 1142-1150.

[4] Institute of Physics and Engineering in Medicine, IPEM Report 78, Catalogue of Diagnostic X-Ray Spectra \& Other Data, (1997).

[5] J. H. Hubbell and S. M. Seltzer, Tables of X-Ray Mass Attenuation Coefficients and Mass Energy-Absorption Coefficients (version 1.4), Online available: www.nist.gov. National Institute of Standards and Technology (NIST), Gaithersburg, MD, (2004).

[6] P. C. Hansen, Regularization tools Version 3.0 for Matlab 5.2, Numer. Algorithms 20 (1999), pp. 195-196. 